\title{
Caution is warranted in using cephamycin antibiotics against recurrent Clostridioides difficile infection
}

\author{
Mark H. Wilcox『 \\ ARISING FROM Y. N. Srikhanta et al. Nature Microbiology https://doi.org/10.1038/s41564-019-0519-1 (2019)
}

In their Article, Srikhanta et al. ${ }^{1}$ suggest that cephamycin antibiotics can be used to treat recurrent Clostridioides difficile infection (CDI) through the inhibition of sporulation. We are concerned that, on the basis of existing data, the use of cephamycins for CDI may not be appropriate.

C. difficile is an important nosocomial pathogen, which is selected by antibiotics that inhibit the gut microbiota. It causes a range of clinical presentations (CDIs) that are associated with high rates of recurrence. CDI recurrence is linked to the colonic survival and persistence of $C$. difficile spores despite antibiotic treatment. The identification of therapeutics that inhibit sporulation is therefore of clinical importance.

Srikhanta et al. propose that cephamycins can be implemented as an adjunct to vancomycin to treat fulminant and recurrent CDIs. However, this conflicts with previous clinical studies implicating cephamycin use as an independent risk factor for the development of $\mathrm{CDIs}^{2-6}$. Cephamycin administration may also lead to severe disruption of the gastrointestinal microbiota ${ }^{7}$ as a consequence of the marked inhibitory effect that cephamycins can have on gut bacteria. Indeed, the cephamycin cefoxitin is used in media in clinical and research settings to selectively culture $C$. difficile from patient samples. Perturbation of the microbiota caused by cephamycins may therefore leave patients susceptible to infection by other bacterial pathogens. A previous study showing that the administration of cefoxitin in human subjects was associated with increases in drug-resistant bacteria and faecal $\beta$-lactamase content in comparison with other antibiotics ${ }^{8}$ is in agreement with this, alongside another study that found overgrowth of enterococci in subjects given cefoxitin'.

Mouse studies have also shown that cefoxitin can promote growth of $C$. difficile and its toxin production in the murine gastrointestinal tract ${ }^{10}$, and that administration of cefotetan results in persistent and high-level gut colonization by vancomycin-resistant enterococci ${ }^{11}$. The use of cephamycins in patients with CDI could therefore exacerbate the symptoms of disease and leave patients susceptible to gastrointestinal colonization by nosocomial pathogens such as vancomycin-resistant enterococci and carbapenemresistant enterobacteria, which are major infection control threats that are difficult to treat and associated with poor patient outcomes.

I contend that the use of cephamycins to treat patients with CDI could lead to adverse patient outcomes. Thus, I caution that the suggestion that "this study could directly and immediately affect treatment of $C$. difficile infection" is premature based on clinical experience with cephamycins. It is therefore imperative that if the observed effects are indeed reproducible in animal models, cephamycins should be tested through formal early-phase human trials before proceeding to appropriately controlled clinical trials designed to assess efficacy and, importantly, adverse effects of cephamycins in combination with vancomycin for the treatment of recurrent and fulminant CDIs. These studies should also actively monitor the impacts that this broad-spectrum antimicrobial combination therapy have on the human gastrointestinal microbiota to determine the extent to which it would leave already vulnerable patients susceptible to potentially serious nosocomial infections.

Received: 22 October 2019; Accepted: 28 November 2019; Published online: 27 January 2020

\section{References}

1. Srikhanta, Y. N. et al. Cephamycins inhibit pathogen sporulation and effectively treat recurrent Clostridioides difficile infection. Nat. Microbiol. 4, 2237-2245 (2019).

2. Carignan, A. et al. Risk of Clostridium difficile infection after perioperative antibacterial prophylaxis before and during an outbreak of infection due to a hypervirulent strain. Clin. Infect. Dis. 46, 1838-1843 (2008).

3. Block, B. S. et al. Clostridium difficile-associated diarrhea follows perioperative prophylaxis with cefoxitin. Am. J. Obstet. Gynecol. 153, 835-838 (1985).

4. Kent, K. C. et al. The impact of Clostridium difficile on a surgical service: a prospective study of 374 patients. Ann. Surg. 227, 296-301 (1998).

5. Crabtree, T. D. et al. Clinical characteristics and antibiotic utilization in surgical patients with Clostridium difficile-associated diarrhea. Am. Surgeon 65, 507-511 (1999).

6. Turner, M. C. et al. Multidisciplinary approach to Clostridium difficile infection in adult surgical patients. J. Am. Coll. Surgeons 228, 570-580 (2019).

7. Stiefel, U. et al. Effect of the increasing use of piperacillin/tazobactam on the incidence of vancomycin-resistant enterococci in four academic medical centers. Infect. Cont. Hosp. Epidemiol. 25, 373-379 (2004).

8. Barza, M. et al. Effect of broad-spectrum parenteral antibiotics on "colonization resistance" of intestinal microflora of humans. Antimicrob. Agents Chemother. 31, 723-727 (1987).

9. Giuliano, M. et al. Effect of broad-spectrum parenteral antibiotics on composition of intestinal microflora of humans. Antimicrob. Agents Chemother. 31, 202-206 (1987).

10. Nerandzic, M. M. \& Donskey, C. J. Effect of ceftobiprole treatment on growth of and toxin production by Clostridium difficile in cecal contents of mice. Antimicrob. Agents Chemother. 55, 2174-2177 (2011).

11. Rice, L. B. et al. $\beta$-lactam antibiotics and gastrointestinal colonization with vancomycin-resistant enterococci. J. Infect. Dis. 189, 1113-1118 (2004).

\section{Competing interests}

M.H.W. has received research funding and/or consultancy funding from several companies developing CDI treatment/prevention therapeutics, including Astellas, Da Volterra, Merck, Pfizer, Sanofi-Pasteur, Seres, Summit, Synthetic Biologics, Valneva and Vaxxilon.

\section{Additional information}

Correspondence and requests for materials should be addressed to M.H.W.

Reprints and permissions information is available at www.nature.com/reprints.

Publisher's note Springer Nature remains neutral with regard to jurisdictional claims in published maps and institutional affiliations.

(C) The Author(s), under exclusive licence to Springer Nature Limited 2020 